\title{
Including birefringence into time evolution of CMB: Current and future constraints
}

\section{Matteo Martinelli*}

Institute for Theoretical Physics, University of Heidelberg

E-mail: martinelli@thphys.uni-heidelberg.de

\begin{abstract}
We introduce birefringence effects within the propagation history of $\mathrm{CMB}$, considering the two cases of a constant and of a linearly incrasing rotation angle. Both cases result into a mixing of $\mathrm{E}$ and $\mathrm{B}$ modes before lensing effects take place, thus leading to the fact that lensing is acting on spectra that are already mixed because of birefringence. After a discussion on the constraints on this effect achieved by state of the art CMB data and the bounds which can be obtained observing also parity violating modes, we focus on degeneracies between polarization rotation and gravitational lensing of CMB photons, which can possibly lead to false detection of non standard lensing effects if birefringence effects are neglected.
\end{abstract}

Proceedings of the Corfu Summer Institute 2015 "School and Workshops on Elementary Particle Physics and Gravity"

1-27 September 2015

Corfu, Greece

\footnotetext{
* Speaker.
} 


\section{Introduction}

Recent Cosmic Microwave Background (CMB) observations brought to more and more precise measurements of temperature anisotropies reaching the almost cosmic variance-limited sensitivity of Planck $[1,2]$. Together with the increased sensitivity in temperature anisotropies, other $\mathrm{CMB}$ experiment were designed in order to measure the $\mathrm{CMB}$ photons polarization properties, such as WMAP [3], QUIET [4] and BICEP[5, 6, 7]. Upcoming surveys are now designed to achieve even more precise measurements of E-modes and to finally detect the parity-odd modes (B modes) of CMB polarization (see e.g. ACTpol[8], SPTpol[9], PolarBear[10] and EBEX[11]).

These observations are crucial to detect signatures of the current standard cosmological model such as the $\mathrm{B}$ modes induced by primordial gravitational waves and the leakage of power between $\mathrm{E}$ and $\mathrm{B}$ modes due to weak gravitational lensing of CMB photons.

Moreover, the precise measurement of $\mathrm{CMB}$ polarization allows also tests of new physics, such as the search for CPT and Lorentz violations in the photons sector of particle physics [12]. In the last few years, this increased polarization sensitivity has allowed to search for signals of birefringence, i.e. rotation of the photons polarization direction during in vacuo propagation (see e.g. $[6,13,14,15,16,17,18,19,20,21,22,23,24,25]$ and references therein), which leads to a mixing between $\mathrm{E}$ and $\mathrm{B}$ polarization modes.

A similar mixing is produced on CMB polarization by weak gravitational lensing and as upcoming surveys will improve our knowledge of this effect, it is crucial to investigate the interplay between these phenomena. While CMB lensing performs its mixing at "recent" times, birefringence starts to take place right after recombination and it is expected to accumulate during photons propagation. Therefore the CMB spectra which are modified by lensing do not encode only the effect of primordial anisotropies, but already contain the rotation effect due to birefringence.

Furthermore, as birefringence modifies also the power spectrum of $B$ modes, giving it an additional contribution due to the leakage from the $E$ modes, it is interesting to investigate the possible degeneracies between birefringence parameters and $\mathrm{CMB}$ lensing. Indeed lensing effect on $\mathrm{CMB}$ spectra, parametrized by the lensing amplitude $A_{L}$ [26], also leads to a leakage from $E$ to $B$ modes, so neglecting the presence of birefringence can in principle produce a misleading detection of a non standard lensing effect $\left(A_{L} \neq 1\right)$.

\section{Cosmic Birefringence}

Cosmic birefringence is the rotation of linear polarization direction during the propagation of radiation in vacuo; this is usually formalized in the literature as a sudden rotation of the polarization after photon propagates from a source to us. From now on we will refer to birefringence effect acting on CMB photons as this is the farthest source we are able to observe.

If the polarization direction rotates counterclockwise (looking at the source) of an angle $\beta>0$, then the Stokes parameters $Q$ and $U$, defined in the standard frame used for CMB [27, 28], get mixed as

$$
\begin{aligned}
& Q=\tilde{Q} \cos 2 \beta+\tilde{U} \sin 2 \beta \\
& U=\tilde{U} \cos 2 \beta-\tilde{Q} \sin 2 \beta
\end{aligned}
$$


and as a consequence $\mathrm{CMB}$ power spectra become

$$
\begin{aligned}
& C_{\ell}^{E E}=\tilde{C}_{\ell}^{E E} \cos ^{2}(2 \beta)+\tilde{C}_{\ell}^{B B} \sin ^{2}(2 \beta)-\tilde{C}_{\ell}^{E B} \sin (4 \beta) \\
& C_{\ell}^{B B}=\tilde{C}_{\ell}^{E E} \sin ^{2}(2 \beta)+\tilde{C}_{\ell}^{B B} \cos ^{2}(2 \beta)+\tilde{C}_{\ell}^{E B} \sin (4 \beta) \\
& C_{\ell}^{E B}=\frac{1}{2}\left(\tilde{C}_{\ell}^{E E}-\tilde{C}_{\ell}^{B B}\right) \sin (4 \beta)+\tilde{C}_{\ell}^{E B}\left(\cos ^{2}(2 \beta)-\sin ^{2}(2 \beta)\right) \\
& C_{\ell}^{T E}=\tilde{C}_{\ell}^{T E} \cos (2 \beta)-\tilde{C}_{\ell}^{T B} \sin (2 \beta) \\
& C_{\ell}^{T B}=\tilde{C}_{\ell}^{T E} \sin (2 \beta)+\tilde{C}_{\ell}^{T B} \cos (2 \beta)
\end{aligned}
$$

Here we indicate with $\tilde{C}_{\ell}$ the spectra in absence of polarization rotation, while the $C_{\ell}$ are the observed spectra. We allow in these equations for the presence of non zero parity-violating crosscorrelation spectra before the rotation occurs, $\tilde{C}_{\ell}^{E B}$ and $\tilde{C}_{\ell}^{T B}$, which in the standard $\Lambda \mathrm{CDM}$ model are vanishing.

However, this treatment of polarization rotation is exact only when considering modification of the spectra happening at the time of observations, i.e. those that one would expect from a systematic miscalibration of the polarimeters [29, 30]. When dealing with birefringence as the effect of some new physics phenomenon instead, eqs. (2.2) can only be considered as an approximation. This comes from the fact that birefringence is a phenomenon due to anomalous photon propagation $[17,18,19,31,32,33]$, thus it accumulates during time. It is therefore necessary to consider the amount of rotation as time dependent, given by $\alpha(\eta)$ as a function of conformal time from last scattering $\eta$.

For a time-dependent rotation of polarization direction, equation (2.1) is easily generalized as

$$
\begin{aligned}
& Q(\eta)=\tilde{Q}(\eta) \cos 2 \alpha(\eta)+\tilde{U}(\eta) \sin 2 \alpha(\eta) \\
& U(\eta)=\tilde{U}(\eta) \cos 2 \alpha(\eta)-\tilde{Q}(\eta) \sin 2 \alpha(\eta)
\end{aligned}
$$

This induces a modification of the Boltzmann equation for the evolution of polarization perturbations, $\Delta_{Q \pm i U}(\vec{k}, \eta)$; moving to the $E$ and $B$ polarization modes for CMB, this leads to [34]

$$
\begin{aligned}
& \Delta_{E, \ell}^{(S)}\left(k, \eta_{0}\right)=\sqrt{\frac{\ell+2}{\ell-2}} \int_{0}^{\eta_{0}} d \eta S_{E}^{(S)}(k, \eta) \cos (2 \delta \alpha(\eta)) j_{\ell}\left(k\left(\eta_{0}-\eta\right)\right) \\
& \Delta_{B, \ell}^{(S)}\left(k, \eta_{0}\right)=\sqrt{\frac{\ell+2}{\ell-2}} \int_{0}^{\eta_{0}} d \eta S_{E}^{(S)}(k, \eta) \sin (2 \delta \alpha(\eta)) j_{\ell}\left(k\left(\eta_{0}-\eta\right)\right) \\
& \Delta_{E, \ell}^{(T)}\left(k, \eta_{0}\right)=\int_{0}^{\eta_{0}} d \eta\left[S_{E}^{(T)}(k, \eta) \cos (2 \delta \alpha(\eta))-S_{B}^{(T)}(k, \eta) \sin (2 \delta \alpha(\eta))\right] j_{\ell}\left(k\left(\eta_{0}-\eta\right)\right) \\
& \Delta_{B, \ell}^{(T)}\left(k, \eta_{0}\right)=\int_{0}^{\eta_{0}} d \eta\left[S_{B}^{(T)}(k, \eta) \cos (2 \delta \alpha(\eta))+S_{E}^{(T)}(k, \eta) \sin (2 \delta \alpha(\eta))\right] j_{\ell}\left(k\left(\eta_{0}-\eta\right)\right)
\end{aligned}
$$

where $S_{E}^{(S, T)}$ and $S_{B}^{(T)}$ are the sources for $E$ and $B$ modes respectively as they appear in eqs. (18) and (30) of [28] and $\delta \alpha(\eta) \equiv \alpha(\eta)-\alpha\left(\eta_{0}\right)=\int_{\eta_{0}}^{\eta} \dot{\alpha}\left(\eta^{\prime}\right) d \eta^{\prime}$. 
Once these relations are obtained, it is possible to compute CMB power spectra in the standard way:

$$
C_{\ell}^{X Y}=(4 \pi)^{2} \int d k k^{2} P_{\phi}(k) \Delta_{X, \ell}\left(k, \eta_{0}\right) \Delta_{Y, \ell}^{*}\left(k, \eta_{0}\right)
$$

Here $P_{\phi}(k)$ is the initial power spectrum and $\Delta_{X, \ell}\left(k, \eta_{0}\right)$ is the perturbation of the mode $X=$ $\{T, E, B\}$ in Fourier space at time $\eta_{0}$, where the temperature term $\Delta_{T, \ell}\left(k, \eta_{0}\right)$ is standard and can be found in [28].

In the following we will focus on a linear time dependence, parameterized as

$$
\alpha(\eta)=\alpha_{1} \frac{\eta}{\eta_{0}}
$$

which leads to $\delta \alpha(\eta)=\alpha_{1} \frac{\eta}{\eta_{0}}-\alpha_{1}$.

This particular time dependence is motivated by quantum-gravity studies [33, 18, 19, 23], leading to an amount of rotation depending quadratically on the energy of the photons and linearly on the propagation time.

Figure 1 shows $\mathrm{CMB}$ polarization spectra with a non vanishing birefringence angle, compared with standard $\Lambda \mathrm{CDM}$, accounting for different possible rotations: a sudden rotation acting on timeevolved spectra (i.e. the one described by the parameter $\beta$ in Eqs. (2.2)), a time dependent rotation which evolves linearly with time and parametrized as in Eq. (2.9), a costant rotation acting on time-evolved spectra before the effect of gravitational lensing is included in CMB polarization.

Accounting correctly for $\mathrm{CMB}$ lensing is crucial when dealing with birefringence; this is due to the fact that, except for the $\beta$ case, lensing acts on the spectra that were rotated by the timeevolving birefringence effect. The interplay of these two phenomena which is included in Figure 1 is described in detail in [34].

As shown in Figure 1, introducing a polarization rotation angle leads to a transfer of power from the $E E$ spectrum to the other polarization modes, which produces non vanishing $C_{\ell}^{T B}$ and $C_{\ell}^{E B}$ and affects the $C_{\ell}^{B B}$ spectrum.

The latter effect is of particular interests as a similar tranfer of power from $E E$ to $B B$ is produced by gravitational lensing of CMB photons; parametrizing the impact of lensing on $B B$ with the lensing amplitude $A_{L}$, which is equal to 1 in a standard $\Lambda C D M$ cosmology, it is possible to infer a degeneracy between lensing and polarization rotation, as the effects brought by birefringence can be (partially) mimicked by an enhanced lensing amplitude (see Figure 2).

\section{Constraints on polarization rotation}

In [34] the birefringence effect is constrained using WMAP latest release [3] and BICEP 2013 release [5] available at the time, exploiting MCMC technique through the publicly available package cosmomc [35] to sample a baseline parameter space composed by standard parameters, namely the baryon and CDM physical matter density $\Omega_{b} h^{2}$ and $\Omega_{c} h^{2}$, the scalar spectral index $n_{s}$, the optical depth $\tau$, the scalar amplitude as evaluated at a pivot scale $k=0.002 \mathrm{Mpc}^{-1} A_{s}$ the angular size of the sound horizon at last scattering surface $\theta$. Furthermore, the parameters describing the birefringence effect are included, i.e. the two constant rotation models discussed above, 

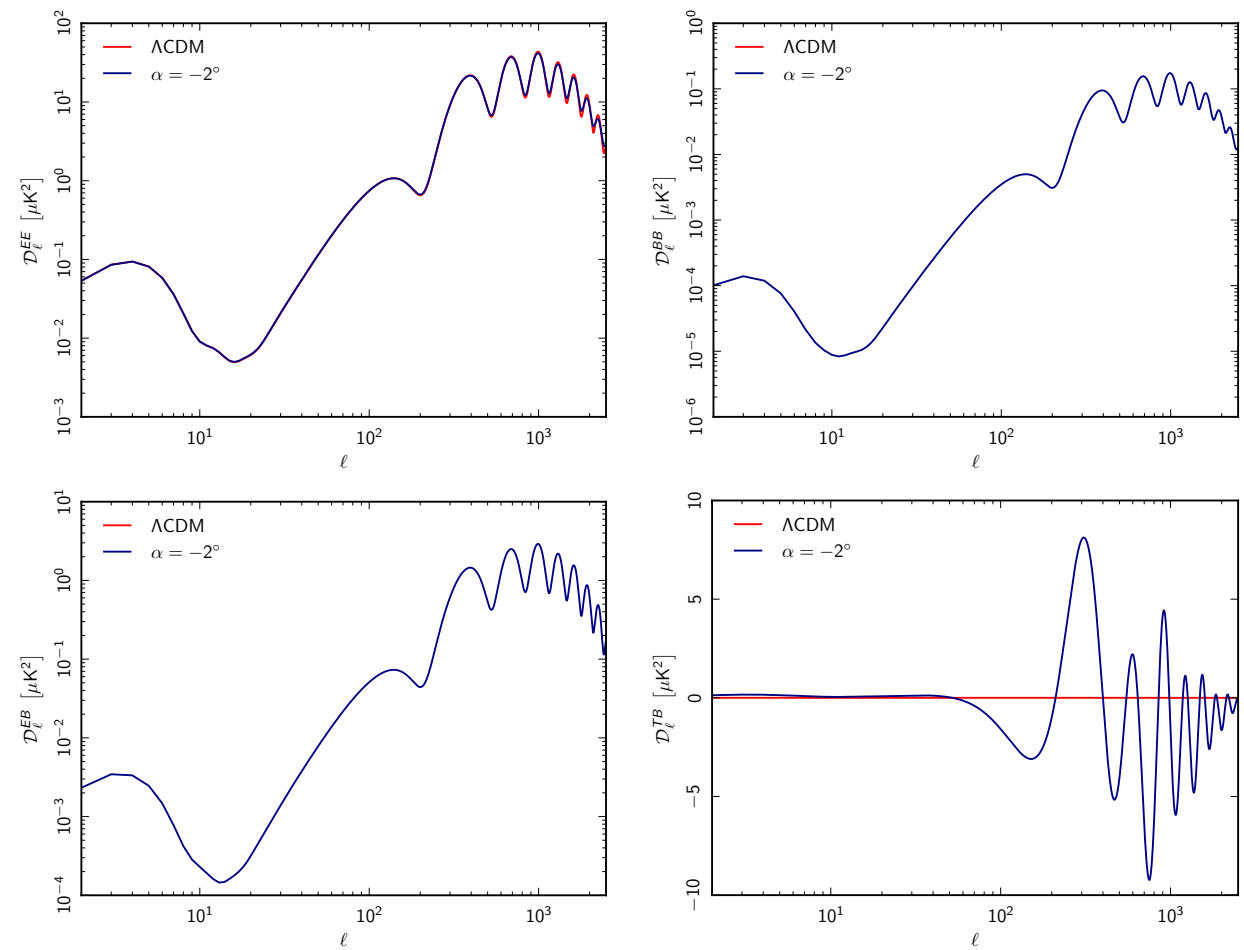

Figure 1: $C M B$ power spectra obtained in standard $\Lambda C D M$ (red solid line), late time constant rotation (pink dotted line), early time constant rotation (green dashed line) and time evolving rotation (blue dashed line) cosmologies. $D_{\ell}=\ell(\ell+1) C_{\ell} / 2 \pi$.

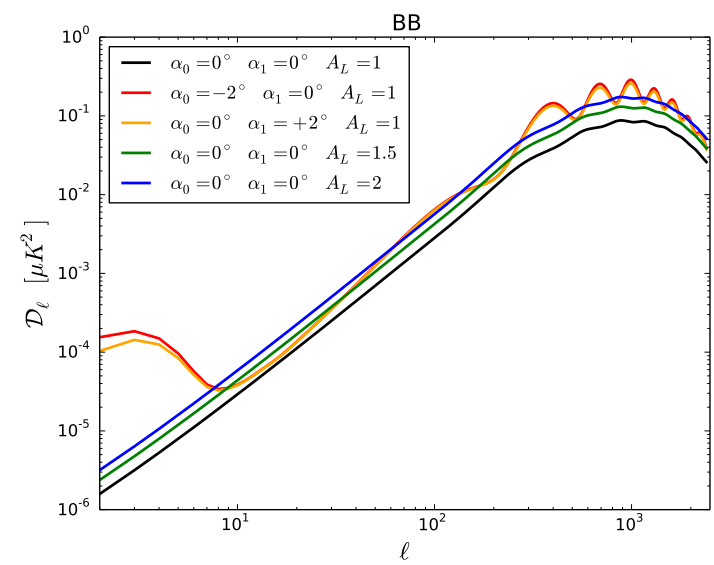

Figure 2: $\mathrm{BB}$ spectra for $\Lambda \mathrm{CDM}$ (black line), rotation cosmologies with a non zero $\alpha_{1}$ (orange line) and $\alpha_{0}$ (red line), compared with the spectra produced by cosmologies without rotation, but with $A_{L}=1.5$ (green line) and $A_{L}=2$ (blue line). $D_{\ell}=\ell(\ell+1) C_{\ell} / 2 \pi$.

parametrized by $\alpha_{0}$ (early times rotation) and $\beta$ (late times rotation) and the parameter describing the time-varying rotation $\alpha_{1}$.

The obtained results [34] are then used as a fiducial cosmology to generate forecasted Planck [36] 
and PolarBear [10] dataset, in order to investigate the performance of these two experiments. These dataset are analyzed following the same procedure described above, obtaining the results shown in Figure 3; these results show how the combination of Planck and Polarbear can significantly distinguish a non vanishing polarization rotation from the standard model.

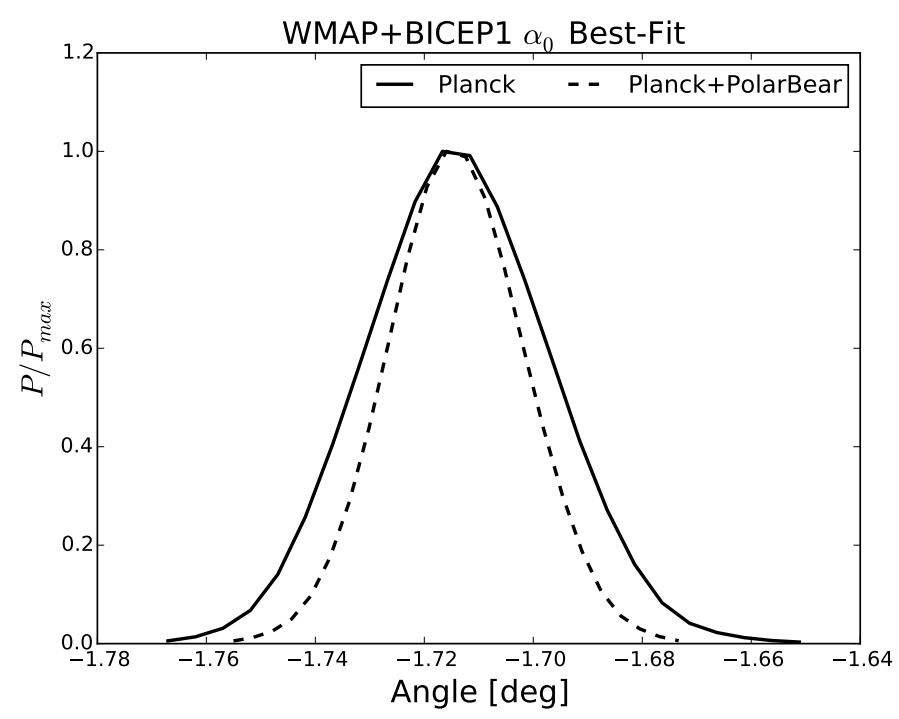

Figure 3: Constraints on the early time constant rotation angle scenario using forecasted Planck and PolarBear datasets.

Following the release of Plank data [37], we performed the analysis on the real CMB spectra; the results of this analysis are shown in Figure 4 and are compatible with what is found in [38]. It is possible to notice how the constrsaints obtained through the forecasted data are not reached when the real Planck data are considered, even including the BICEP-Keck-Planck (BKP) data combination for $E E$ and $B B$ spectra [39]; this is due to the fact that the 2015 Planck release did not made available the $T B$ and $E B$ spectra (included in the forecasted datasets used in [34]), which are crucial to improve the constraints and distinguish a positive angle from a negative one.

\section{Birefringence and CMB lensing}

As discussed in Section 2 and shown in Figure 2, we expect that the birefringence angle will be degenerate with a varying CMB lensing amplitude $A_{L}$. This is of particular interest given the results obtained on $A_{L}$ by the Planck collaboration [40] where the best fit value of this parameter is departing from the $\Lambda \mathrm{CDM}$ expectation when the $\mathrm{CMB}$ temperature and polarization spectra are analyzed, while exploiting CMB lensing extraction tools leads to results compatible with the standard $A_{L}=1$ (see Figure 5). 


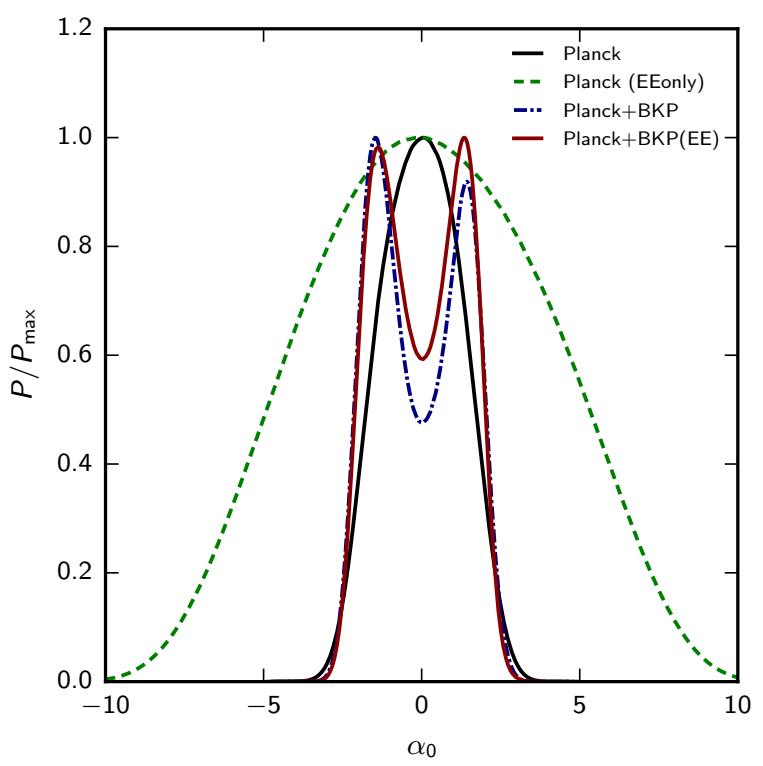

Figure 4: Constraints on the early time constant rotation scenario obtained with Planck 2015 data, using polatization data alone (green dashed line) and both temperature and polarization (black solid line). Planck data are also combined with the data obtained by the BKP joint analys, considering only $B$ modes (red solid line) and both $B$ and $E$ modes (blue dot-dashed line).

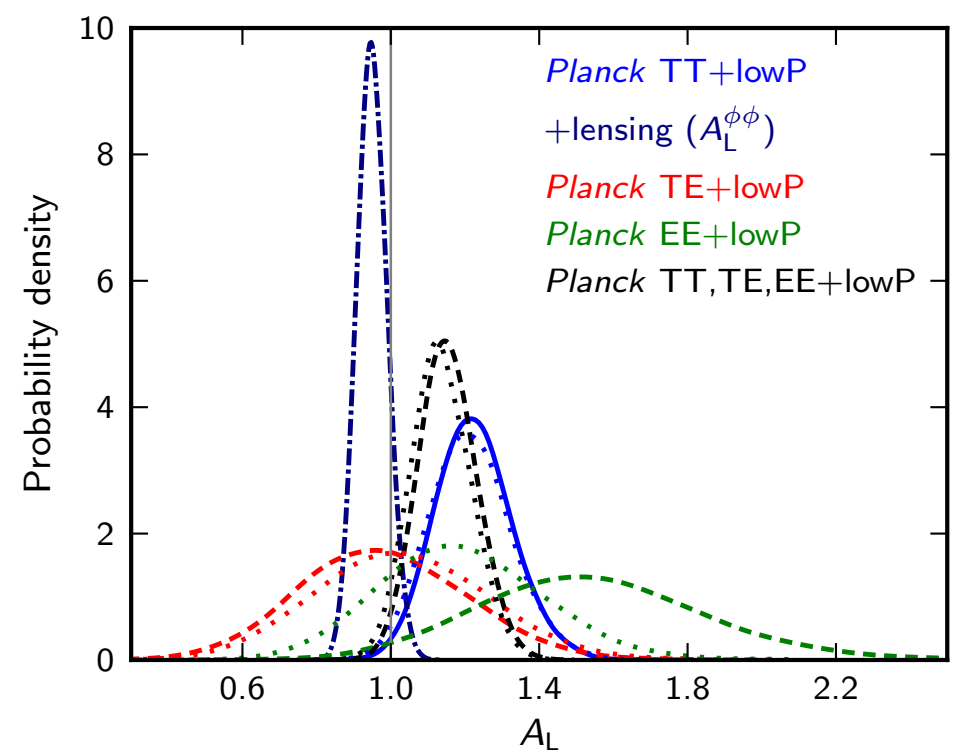

Figure 5: Lensing amplitude obtained with different combination of Planck data [40].

We found in [34] that a non standard value of $A_{L}$ is obtained when a forecasted dataset which includes polarization rotation is analyzed neglecting this phenomenon; Figure 6 shows the results 
obtained analyzing the forecasted data described above assuming three different cosmologies: the first includes the birefringence effect, the second is a standard $\Lambda \mathrm{CDM}$ and the third does not include polarization rotation, but allows for a varying lensing amplitude $A_{L}$.

While the results obtained with the first assumption (black lines) recover the fiducial values of the cosmological parameters, the results of the second analysis (red lines) are biased because of the inability of a $\Lambda \mathrm{CDM}$ model to recover the fiducial CMB power spectra. Interestingly, the inclusion of a varying $A_{L}$ (blue lines) mitigates the shift produced assuming the wrong cosmological model. This is due to the fact that raising the value of $A_{L}$ increases the power transfer from $E E$ to $B B$ modes and therefore can partially account for enhancement in the $B B$ spectrum produced by birefringence when the datasets are analyzed assuming vanishing rotation angles. It is interesting to point out how the shift in the standard parameters is mitigated at the price of a non standard value of $A_{L}=1.29 \pm 0.03$; this means that neglecting the presence of a birefringence rotation can possibly lead to a false detection of $A_{L}>1$ when analyzing data with Planck+PolarBear sensitivity.
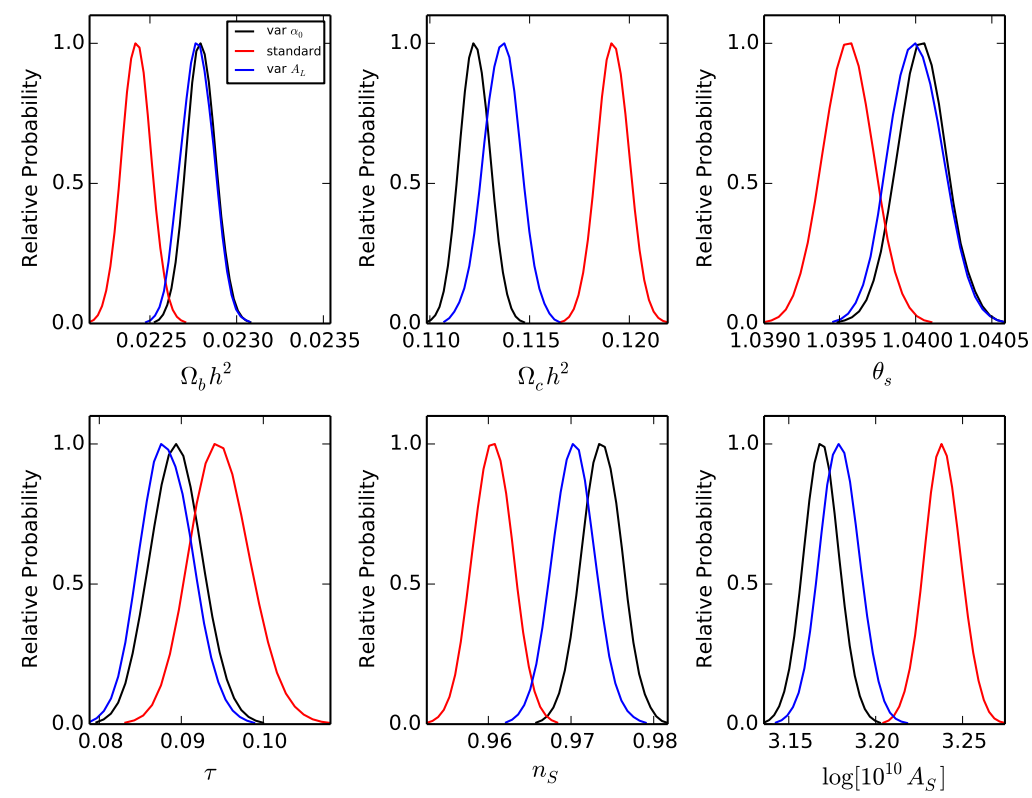

Figure 6: Posterior distribution for the parameters using Planck forecasted datasets, with a fiducial cosmology which includes polarization rotation, analyzed with a varying $\alpha_{0}$ (black lines), with a varying $A_{L}$ (blue lines) and with only standard cosmological parameters (red lines). One sees that $A_{L}$ can compensate for the effects of birefringence quite well in experiments with Planck sensitivity.

\section{Discussion}

In this paper we discussed the results obtained in [34], where, using forecasted Planck and PolarBear data we obtained constraints on several polarization rotation scenarios; the results highlight 
how current and upcoming CMB surveys will be able to distinguish cosmologies which include birefringence from the standard $\Lambda \mathrm{CDM}$ model.

The analysis of Planck 2015 and BKP data show instead the relevance of the inclusion of parity violating mode data; when these are not included in the analysis it is in fact not possible to distinguish between different signs of the rotation angle, as shown in Figure 4.

Furthermore, we focused on the degeneracy that birefringence parameters have with CMB lensing amplitude $A_{L}$; in particular we showed that analyzing datasets which includes polarization rotation with a cosmology which neglects birefringence can lead to a false detection of a non standard $A_{L}$, as an enhanced lensing amplitude is able to partially mimic the power leakage from $E$ to $B$ modes brought by birefringence. It will be therefore interesting to further investigate the interplay between lensing and polarization rotation as it can possibly mitigate the discrepancy found by the Planck collaboration between the values of $A_{L}$ measured from anisotropies spectra and through CMB lensing reconstruction.

\section{Acknowledgments}

MM acknowledges the DFG TransRegio TRR33 grant on The Dark Universe.

\section{References}

[1] P. A. R. Ade et al. [Planck Collaboration], arXiv:1303.5062 [astro-ph.CO].

[2] P. A. R. Ade et al. [Planck Collaboration], Astron. Astrophys. (2014) [arXiv:1303.5076 [astro-ph.CO]].

[3] Bennett, C. L., Larson, D., Weiland, J. L., et al. 2013, ApJS, 208, 20

[4] QUIET Collaboration, Araujo, D., Bischoff, C., et al. 2012, ApJ, 760, 145

[5] D. Barkats et al. [BICEP1 Collaboration], arXiv:1310.1422 [astro-ph.CO].

[6] Kaufman, J. P., Miller, N. J., Shimon, M., et al. 2014, Phys. Rev. D, 89, 062006

[7] P. A. R. Ade et al. [BICEP2 Collaboration], Phys. Rev. Lett. 112 (2014) 241101 [arXiv:1403.3985 [astro-ph.CO]].

[8] Niemack, M. D., Ade, P. A. R., Aguirre, J., et al. 2010, Proc. SPIE , 7741,

[9] Austermann, J. E., Aird, K. A., Beall, J. A., et al. 2012, Proc. SPIE , 8452,

[10] P. A. R. Ade et al. [POLARBEAR Collaboration], Phys. Rev. Lett. 112 (2014) 131302

[arXiv:1312.6645 [astro-ph.CO]].

[11] MacDermid, K., Aboobaker, A. M., Ade, P., et al. 2014, arXiv:1407.6894

[12] B. Feng, M. Li, J. Q. Xia, X. Chen and X. Zhang, Phys. Rev. Lett. 96 (2006) 221302 [astro-ph/0601095].

[13] A. Lue, L. M. Wang and M. Kamionkowski, Phys. Rev. Lett. 83, 1506 (1999) [arXiv:astro-ph/9812088].

[14] N. F. Lepora, arXiv:gr-qc/9812077. 
[15] V. A. Kostelecky and M. Mewes, Phys. Rev. D 66 (2002) 056005 [arXiv:hep-ph/0205211].

[16] J. Q. Xia, H. Li, X. 1. Wang and X. m. Zhang, Astron. Astrophys. 483 (2008) 715 [arXiv:0710.3325 [hep-ph]].

[17] F. Finelli and M. Galaverni, Phys. Rev. D 79 (2009) 063002 [arXiv:0802.4210 [astro-ph]].

[18] T. Kahniashvili, R. Durrer and Y. Maravin, Phys. Rev. D 78 (2008) 123009 [arXiv:0807.2593 [astro-ph]].

[19] G. Gubitosi, L. Pagano, G. Amelino-Camelia, A. Melchiorri and A. Cooray, arXiv:0904.3201 [astro-ph.CO].

[20] P. Cabella, P. Natoli \& J. Silk, 2007, prd, 76, 123014

[21] K. R. S. Balaji, R. H. Brandenberger and D. A. Easson, JCAP 0312, 008 (2003) [arXiv:hep-ph/0310368].

[22] A. Gruppuso, P. Natoli, N. Mandolesi, A. De Rosa, F. Finelli and F. Paci, JCAP 1202 (2012) 023 [arXiv:1107.5548 [astro-ph.CO]].

[23] G. Gubitosi and F. Paci, JCAP 1302 (2013) 020 [arXiv:1211.3321 [astro-ph.CO]].

[24] M. Giovannini and K. E. Kunze, Phys. Rev. D 79 (2009) 087301 [arXiv:0812.2804 [astro-ph]].

[25] A. P. S. Yadav, M. Shimon and B. G. Keating, Phys. Rev. D 86 (2012) 083002 [arXiv:1207.6640 [astro-ph.CO]].

[26] E. Calabrese, A. Slosar, A. Melchiorri, G. F. Smoot and O. Zahn, Phys. Rev. D 77 (2008) 123531 [arXiv:0803.2309 [astro-ph]].

[27] G. Hinshaw et al. [WMAP Collaboration], Astrophys. J. Suppl. 180 (2009) 225 [arXiv:0803.0732 [astro-ph]].

[28] M. Zaldarriaga and U. Seljak, Phys. Rev. D 55 (1997) 1830 [astro-ph/9609170].

[29] L. Pagano, P. de Bernardis, G. De Troia, G. Gubitosi, S. Masi, A. Melchiorri, P. Natoli and F. Piacentini et al., Phys. Rev. D 80 (2009) 043522 [arXiv:0905.1651 [astro-ph.CO]].

[30] J. Kaufman, B. Keating and B. Johnson, arXiv:1409.8242 [astro-ph.CO].

[31] S. M. Carroll, G. B. Field and R. Jackiw, Phys. Rev. D 41 (1990) 1231.

[32] G. C. Liu, S. Lee and K. W. Ng, Phys. Rev. Lett. 97 (2006) 161303 [arXiv:astro-ph/0606248].

[33] R. C. Myers and M. Pospelov, Phys. Rev. Lett. 90 (2003) 211601 [hep-ph/0301124].

[34] G. Gubitosi, M. Martinelli and L. Pagano, JCAP 1412 (2014) no.12, 020 doi:10.1088/1475-7516/2014/12/020 [arXiv:1410.1799 [astro-ph.CO]].

[35] A. Lewis and S. Bridle, Phys. Rev. D 66 (2002) 103511 [arXiv:astro-ph/0205436].

[36] [Planck Collaboration], "Planck: The scientific programme," arXiv:astro-ph/0604069.

[37] N. Aghanim et al. [Planck Collaboration], [arXiv:1507.02704 [astro-ph.CO]].

[38] A. Gruppuso, M. Gerbino, P. Natoli, L. Pagano, N. Mandolesi and D. Molinari, arXiv:1509.04157 [astro-ph.CO].

[39] P. A. R. Ade et al. [BICEP2 and Planck Collaborations], Phys. Rev. Lett. 114 (2015) 101301 doi:10.1103/PhysRevLett.114.101301 [arXiv:1502.00612 [astro-ph.CO]].

[40] P. A. R. Ade et al. [Planck Collaboration], doi:10.1051/0004-6361/201525941 arXiv:1502.01591 [astro-ph.CO]. 